\title{
Theory for the enhanced induced magnetization in coupled magnetic trilayers in the presence of spin fluctuations
}

\author{
P.J. Jensen, ${ }^{1,2}$ K.H. Bennemann, ${ }^{2}$ \\ ${ }^{1}$ Hahn-Meitner-Institut, Glienicker Str.100, D - 14109 Berlin, Germany \\ ${ }^{2}$ Institut für Theoretische Physik, Freie Universität Berlin \\ Arnimallee 14, D - 14195 Berlin, Germany \\ P. Poulopoulos, M. Farle, ${ }^{*}$ F. Wilhelm, K. Baberschke \\ Institut für Experimentalphysik, Freie Universität Berlin \\ Arnimallee 14, D - 14195 Berlin, Germany
}

(April 11, 2018)

\begin{abstract}
Motivated by recent experiments, the effect of the interlayer exchange interaction $J_{\text {inter }}$ on the magnetic properties of coupled $\mathrm{Co} / \mathrm{Cu} / \mathrm{Ni}$ trilayers is studied theoretically. Here the Ni film has a lower Curie temperature $T_{C, \mathrm{Ni}}$ than the Co film in case of decoupled layers. We show that by taking into account magnetic fluctuations the interlayer coupling induces a strong magnetization for $T \gtrsim T_{C, \mathrm{Ni}}$ in the $\mathrm{Ni}$ film. For an increasing $J_{\text {inter }}$ the resonance-like peak of the longitudinal Ni susceptibility is shifted to larger temperatures, whereas its maximum value decreases strongly. A decreasing $\mathrm{Ni}$ film thickness enhances the induced $\mathrm{Ni}$ magnetization for $T \gtrsim T_{C, \mathrm{Ni}}$. The measurements cannot be explained properly by a mean field estimate, which yields a ten times smaller effect. Thus, the observed magnetic properties indicate the strong effect of $2 \mathrm{D}$ magnetic fluctuations in these layered magnetic systems. The calculations are performed with the help of a Heisenberg Hamiltonian and a Green's function approach.
\end{abstract}

75.10.Jm, 75.30.Ds, 75.70.Cn

Recently, the element specific magnetization and the longitudinal susceptibility of magnetic epitaxial $\mathrm{Co} / \mathrm{Cu} / \mathrm{Ni}$ trilayers grown on $\mathrm{Cu}(001)$ have been measured [1,2]. The two ferromagnetic $\mathrm{Ni}$ and Co films are coupled by the indirect exchange interaction $J_{\text {inter }}$ across the nonmagnetic $\mathrm{Cu}$ layer, which exhibits an oscillatory behavior as a function of the thickness $d_{\mathrm{Cu}}$ of the spacer, and an overall decay like $d_{\mathrm{Cu}}^{-2}$ [2, 4. The thicknesses $d_{\mathrm{Ni}}$ and $d_{\mathrm{Co}}$ of the $\mathrm{Ni}$ and Co films are chosen in such a way that for a vanishing interlayer coupling the Ni film has a lower Curie temperature than the Co film, i.e. $T_{C, \mathrm{Ni}}\left(d_{\mathrm{Ni}}\right)<T_{C, \mathrm{Co}}\left(d_{\mathrm{Co}}\right) . \quad J_{\text {inter }}$ induces for $T>T_{C, \mathrm{Ni}}$ a considerable magnetization in the Ni film, which has been measured to vanish $\sim 30-40 \mathrm{~K}$ above $T_{C, \mathrm{Ni}}$. In this work we show that the induced strong Ni magnetization can theoretically only be obtained properly by taking into account magnetic fluctuations in the $\mathrm{Ni}$ film. If these fluctuations are neglected in the calculations (for example within a mean field theory (MFT) approach 5), the resulting induced $\mathrm{Ni}$ magnetization $M_{\mathrm{Ni}}(T)$ for $T \gtrsim T_{C, \mathrm{Ni}}$ is an order of magnitude smaller. Vice versa, the neglect of these fluctuations requires an unrealistic large value for $J_{\text {inter }}$ to yield the observed shift $\Delta T$ of $M_{\mathrm{Ni}}(T)$ to larger temperatures. Generally, spin fluctuations diminish the magnetization of a two-dimensional (2D) magnetic system more strongly than for bulk magnets [6, [7]. An external magnetic field suppresses the action of these fluctuations, resulting in a stronger increase of the magnetization in 2D than in bulk systems. Similarly, in case of a coupled trilayer the interlayer coupling reduces the fluctuation effect, since it acts as an external magnetic field. Consequently, $J_{\text {inter }}$ has a pronounced effect on the Ni film magnetization. The magnetic behavior of such a system can be used to study the action of the strong $2 \mathrm{D}$ spin fluctuations.

To take into account the collective magnetic excitations (spin waves, magnons), we apply a many-body Green's function approach, and use the so-called Tyablikov (or RPA-) decoupling [8]. Since within this method interactions between magnons are partly taken into account, the whole temperature range of interest up to the Curie temperature can be considered. A Heisenberg-type Hamiltonian on an fcc(001) thin film with $d=d_{\mathrm{Ni}}+d_{\mathrm{Co}}$ monolayers (ML) is assumed with localized magnetic moments $\boldsymbol{\mu}_{i}=\mu_{i} \mathbf{S}_{i} / S$ on lattice sites $i$ :

$$
\begin{aligned}
\mathcal{H}= & -\frac{1}{2} \sum_{\langle i, j\rangle} J_{i j} \mathbf{S}_{i} \mathbf{S}_{j}-\sum_{i} \mathbf{B} \boldsymbol{\mu}_{i} \\
& +\frac{1}{2} \sum_{\substack{i, j \\
i \neq j}} \frac{1}{r^{5}}\left[\boldsymbol{\mu}_{i} \boldsymbol{\mu}_{j} r^{2}-3\left(\mathbf{r} \boldsymbol{\mu}_{i}\right)\left(\mathbf{r} \boldsymbol{\mu}_{j}\right)\right] .
\end{aligned}
$$

Quantum mechanical spins with spin quantum number $S=1$ are assumed. Due to the dipole interaction the magnetization $M_{i}(T)=\left\langle S_{i}^{z}\right\rangle$ is directed in-plane, determining the quantization axis ( $z$-direction). The external magnetic field $\mathbf{B}=(0,0, B)$ is applied parallel to this axis. $J_{i j}$ are the exchange couplings between nearest neighbor spin pairs which are chosen in such a way that they yield the observed Curie temperatures for the separate (i.e. decoupled) layers. We put $J_{\mathrm{CoCo}}=398 \mathrm{~K}$ per bond to obtain $T_{C, \mathrm{Co}}(2)=435 \mathrm{~K}$ for a Co film with $d_{\mathrm{Co}}=2$ ML 11. To account for the diminished interface magnetic state of the Ni film, we distinguish between exchange couplings in the interface layers and the film in- 
terior layers. With $J_{\mathrm{NiNi}}^{\text {interface }}=30 \mathrm{~K}$ and $J_{\mathrm{NiNi}}^{\text {interior }}=172$ $\mathrm{K}$ per bond one obtains for a Ni film with $d_{\mathrm{Ni}}=5 \mathrm{ML}$ $T_{C, \mathrm{Ni}}(5)=267 \mathrm{~K} 2$. These numbers for the exchange couplings are somewhat lower than the corresponding values obtained from the bulk Curie temperatures. In addition an interlayer exchange coupling $J_{\text {inter }}$ across the nonmagnetic $\mathrm{Cu}$ spacer layer between $\mathrm{Ni}$ and $\mathrm{Co}$ spins in the interlayer next to $\mathrm{Cu}$ is assumed. Positive as well as negative values of $J_{\text {inter }}$ can be considered, preferring thus parallel $\left(J_{\text {inter }}>0\right)$ or antiparallel $\left(J_{\text {inter }}<0\right)$ magnetized $\mathrm{Ni}$ and Co layers. The last term in Eq.(1) is the magnetic dipole coupling between spins $\boldsymbol{\mu}_{i}$ and $\boldsymbol{\mu}_{j}$ separated by vectors $\mathbf{r}=\mathbf{r}_{j}-\mathbf{r}_{i}$, denoting $r=|\mathbf{r}|$. The slowly converging oscillating lattice sums are converted into rapidly converging ones with the help of Ewald summation [9]. Layer-dependent magnetic moments $\mu_{i}$ are assumed [10]. In particular we put $\mu_{\mathrm{Ni}}^{\text {interface }}=0.46 \mu_{B}$, $\mu_{\mathrm{Ni}}^{\text {interior }}=0.61 \mu_{B}$, and $\mu_{\mathrm{Co}}=2.02 \mu_{B}$ for all Co layers, $\mu_{B}$ is the Bohr magneton. Lattice anisotropy terms are not considered here.

For the calculation of the layer-dependent magnetizations, $M_{i}(T), i=1 \ldots d$, we consider the following twotimes (commutator-) Green's functions, which are written in spectral representation as 11, 12

$G_{i j}^{+-(n)}\left(\omega, \mathbf{k}_{\|}\right)=\left\langle\left\langle S_{i}^{+} ;\left(S_{j}^{z}\right)^{n} S_{j}^{-}\right\rangle\right\rangle_{\omega, \mathbf{k}_{\|}}=\left\langle\left\langle S_{i}^{+} ; C_{j}^{(n)}\right\rangle\right\rangle_{\omega, \mathbf{k}_{\|}}$.

Here the $i, j$ refer to layer indices. Because we assume ferromagnetically ordered layers, the lateral periodicity has been used to apply a Fourier transformation into the $2 \mathrm{D}$ momentum space, $\mathbf{k}_{\|}$being the $2 \mathrm{D}$ wave vector. The Green's functions are determined by solving the familiar equation of motion. Higher order Green's functions are approximated by the Tyablikov (RPA-) decoupling [8] for the exchange and dipole interactions $(i \neq k)$ :

$$
\left\langle\left\langle S_{i}^{z} S_{k}^{+} ; C_{j}^{(n)}\right\rangle\right\rangle \approx\left\langle S_{i}^{z}\right\rangle\left\langle\left\langle S_{k}^{+} ; C_{j}^{(n)}\right\rangle\right\rangle=M_{i}(T) G_{k j}^{+-(n)},
$$

i.e. spin operators $S_{i}^{z}$ are replaced by their expectation values $M_{i}(T)$. Different integers $n \leq 2 S-1$ have to be considered in order to calculate different spin quantum numbers $S$ [1]. The equations of motion lead to a set of $d$ coupled linear equations for the Green's functions. With the help of the spectral theorem the respective expectation values (or correlation functions) $\left\langle\left(S_{j}^{z}\right)^{n} S_{j}^{-} S_{i}^{+}\right\rangle$are determined. The magnetization $M_{i}(T)$ is obtained from the usual relations between spin operators. By comparison with a recent Quantum Monte Carlo calculation of a Heisenberg monolayer in an external magnetic field 13 it was shown that the applied Green's function method yields a satisfactory result for the magnetization [14,15].

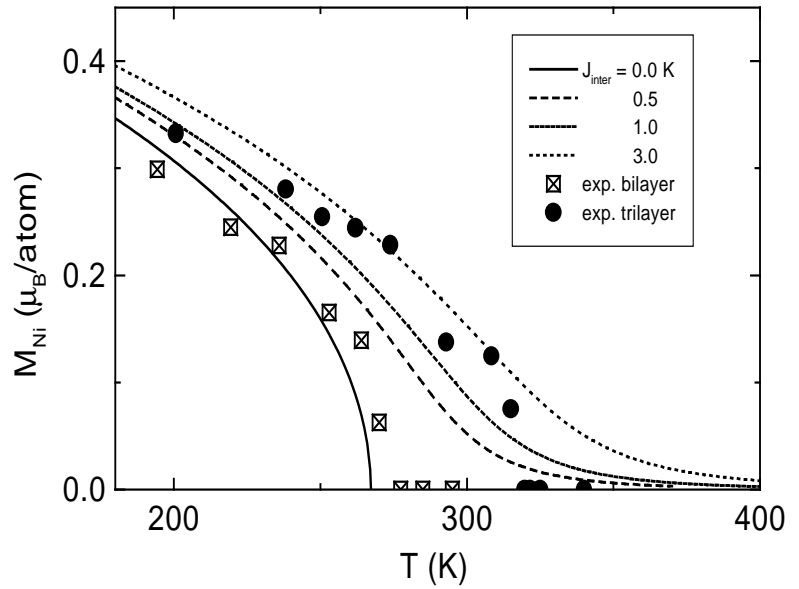

FIG. 1. Ni magnetization $M_{\mathrm{Ni}}(T)$ for a $\mathrm{Co} / \mathrm{Cu} / \mathrm{Ni}$ trilayer as a function of the temperature $T$ calculated by the Green's function approach. Different interlayer couplings $J_{\text {inter }}$ (in $\mathrm{K} /$ bond) are assumed as indicated. An epitaxial trilayer with $d_{\mathrm{Ni}}=5 \mathrm{Ni}$ and $d_{\mathrm{Co}}=3$ Co monolayers (ML) is assumed. The values for the exchange couplings within the $\mathrm{Ni}$ and $\mathrm{Co}$ films and the corresponding magnetic moments are given in the text. $T_{C, \mathrm{Ni}}=267 \mathrm{~K}$ is the Curie temperature of the single Ni film. In addition experimental results for the $\mathrm{Ni}$ magnetization are displayed for a single $4.8 \mathrm{ML}$ Ni film capped with $\mathrm{Cu}$ ('bilayer'), as well as for a $\mathrm{Co} / \mathrm{Cu} / \mathrm{Ni}$ trilayer with $d_{\mathrm{Ni}}=4.8 \mathrm{ML}, d_{\mathrm{Co}}=2.8 \mathrm{ML}$, and $d_{\mathrm{Cu}}=2.8 \mathrm{ML}[2]$.

In Fig.(1) we present results for the Ni magnetization $M_{\mathrm{Ni}}(T)$ as a function of the temperature $T$ calculated with different interlayer couplings $J_{\text {inter }}$. We consider a $\mathrm{Co} / \mathrm{Cu} / \mathrm{Ni}$ trilayer with $d_{\mathrm{Ni}}=5 \mathrm{Ni}, d_{\mathrm{Cu}}=3 \mathrm{Cu}$, and $d_{\mathrm{Co}}=3$ Co monolayers, respectively. For comparison experimental results for the same system are also shown [2]. The layer-dependent magnetizations $M_{i}(T)$, $i=1 \ldots d$, are determined from an iterative procedure. Presented are the $\mathrm{Ni}$ magnetizations $M_{\mathrm{Ni}}(T)$ averaged over all $\mathrm{Ni}$ layers. We use the inflection point $T_{\text {infl }}$ of $M_{\mathrm{Ni}}(T)$ as a measure of the corresponding temperature shift $\Delta T\left(J_{\text {inter }}\right)=T_{\text {infl }}-T_{C, \mathrm{Ni}}$ of the Ni magnetization with respect to the decoupled case. One observes that already a small value of the interlayer coupling $J_{\text {inter }}$ produces a comparably large $\Delta T\left(J_{\text {inter }}\right)$. For example, $J_{\text {inter }}=1 \mathrm{~K}$ results in $\Delta T \approx 30 \mathrm{~K}$. Such a value for $J_{\text {inter }}$ compares well with various results measured formerly with different methods [4, 16]. Corresponding results have been determined by us also from a MFT approach. For the same value of $J_{\text {inter }}$ the calculated $\Delta T\left(J_{\text {inter }}\right)$ obtained from this approximation is about 10 times smaller than the value resulting from the Green's function method.

We stress that this strong difference is a result of the $2 \mathrm{D}$ character of the magnetic trilayer system. The action of an external magnetic field for $T \gtrsim T_{C}$ is much more pronounced for a $2 \mathrm{D}$ magnet than for a corresponding bulk system [7]. For the coupled trilayer system under 
consideration the interlayer coupling $J_{\text {inter }}$ acts similar as an external magnetic field. Therefore, for temperatures $T \gtrsim T_{C, \mathrm{Ni}}$ close to the Curie temperature of the single Ni film already a small $J_{\text {inter }}$ is sufficient to induce a marked Ni magnetization and the corresponding temperature shift $\Delta T$. In contrast, within a MFT approach the exchange coupling alone results in a finite remanent magnetization for a 2D magnet, and does not need the support of the dipole coupling or an external magnetic field. In this case a small interlayer coupling adds simply to the strong $\mathrm{Ni}-\mathrm{Ni}$ exchange coupling and results in a correspondingly small value of $\Delta T$.

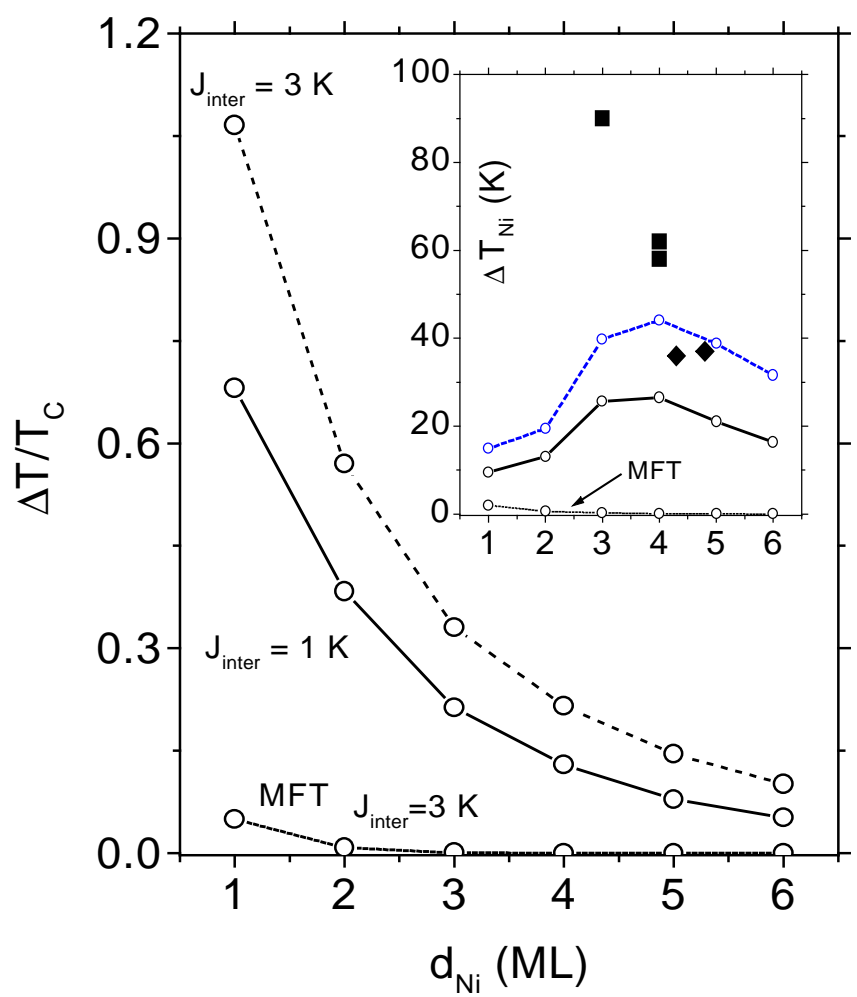

FIG. 2. Temperature difference $\Delta T\left(d_{\mathrm{Ni}}\right)$ between the inflection point of the $\mathrm{Ni}$ magnetization $M_{\mathrm{Ni}}(T)$ in the coupled $\mathrm{Co} / \mathrm{Cu} / \mathrm{Ni}$ trilayer and the Curie temperature $T_{C, \mathrm{Ni}}\left(d_{\mathrm{Ni}}\right)$ of the single $\mathrm{Ni}$ film as a function of the Ni film thickness $d_{\mathrm{Ni}}$. Two different interlayer couplings $J_{\text {inter }}=1 \mathrm{~K}$ and $3 \mathrm{~K}$ are assumed, the other system parameters are the same as for Fig.1. The figure displays the relative temperature difference $\Delta T$ scaled by $T_{C, \mathrm{Ni}}\left(d_{\mathrm{Ni}}\right)$. In the inset the absolute temperature shift is given. For comparison we show the respective results for $\Delta T$ calculated by an MFT approach, assuming $J_{\text {inter }}=3 \mathrm{~K}$. Experimental results are also given for two different $\mathrm{Cu}$ thicknesses: $\bullet d_{\mathrm{Cu}}=2.8 \mathrm{ML}$; and $\mathbf{\square}: d_{\mathrm{Cu}}=2.0$ $\mathrm{ML}$, the latter corresponds to a larger $J_{\text {inter }}>3 \mathrm{~K}$.

We have tested the assumption that the interlayer coupling acts similar as an external magnetic field. The results of $M_{\mathrm{Ni}}(T)$ for a single (decoupled) Ni film with $d_{\mathrm{Ni}}=5 \mathrm{ML}$, with an external magnetic field acting exclusively on the topmost $\mathrm{Ni}$ layer with a strength
$B=J_{\text {inter }} / \mu_{\mathrm{Ni}}$, are practically the same as for the corresponding coupled trilayer system.

Furthermore, we have calculated the induced Ni magnetization at $T \gtrsim T_{C, \mathrm{Ni}}$ for different thicknesses $d_{\mathrm{Ni}}$ of the Ni film. Results for $\Delta T\left(d_{\mathrm{Ni}}\right)$ are shown in Fig.(2) for two different values of $J_{\text {inter }}$ and for $1 \leq d_{\mathrm{Ni}} \leq 6 \mathrm{ML}$. The other coupling parameters are the same. The resulting absolute value $\Delta T\left(d_{\mathrm{Ni}}\right)$ exhibits a maximum at about $d_{\mathrm{Ni}}=4 \mathrm{ML}$, see inset of Fig.(2). For comparison also the corresponding results calculated by the MFT approach are shown. On the other hand, the relative temperature shift $\Delta T / T_{C, \mathrm{Ni}}\left(d_{\mathrm{Ni}}\right)$, scaled by the Curie temperature $T_{C, \mathrm{Ni}}\left(d_{\mathrm{Ni}}\right)$ of the single Ni film, increases by reducing the thickness of the Ni film, s. Fig.(2). This indicates the increasing importance of the action of the magnetic fluctuations for a decreasing film thickness. Experimental results are also displayed for two different $\mathrm{Cu}$ spacer thicknesses.

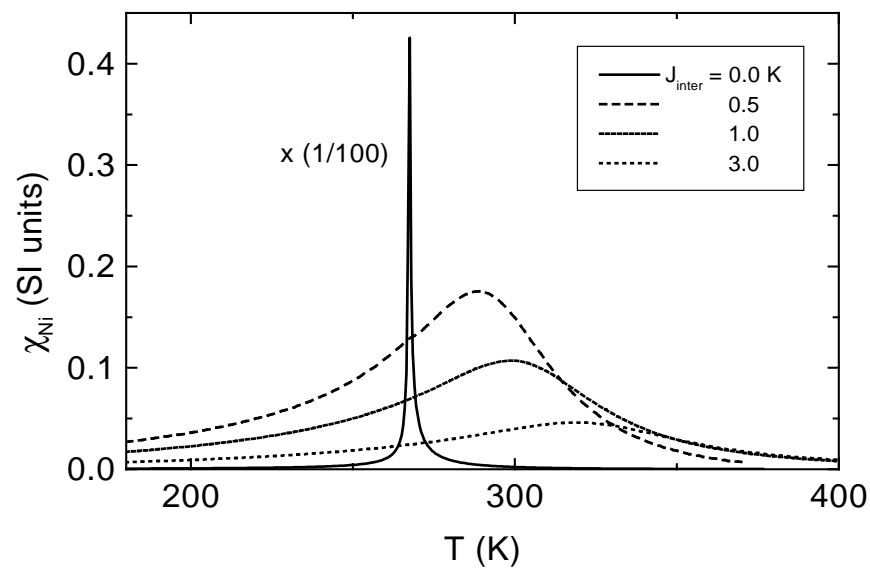

FIG. 3. Ni film susceptibility $\chi_{\mathrm{Ni}}(T)$ as a function of the temperature for different interlayer couplings as indicated. The susceptibility field amplitude is $2 \mathrm{G}$. The same $\mathrm{Co} / \mathrm{Cu} / \mathrm{Ni}$ trilayer system as described in Fig.(1) is assumed. Note that the susceptibility curve for $J_{\text {inter }}=0$ is scaled by the factor $1 / 100$.

In addition we investigate the longitudinal susceptibility $\chi_{\mathrm{Ni}}(T)$ of the Ni film. For this purpose $\chi_{i}(T)$ for the $i$ th magnetic layer, $i=1 \ldots d$, is calculated from the difference of the magnetizations $M_{i}(T, B)$ with $B=0$ and $B=2 \mathrm{G}$,

$$
\chi_{i}^{z z}(T) \equiv \chi_{i}(T)=\frac{\partial M_{i}(T, B)}{\partial B} \approx \frac{\Delta M_{i}(T, B)}{\Delta B} .
$$

In Fig.(3) the Ni susceptibility $\chi_{\mathrm{Ni}}(T)$ averaged over all $\mathrm{Ni}$ layers is displayed, corresponding to the results of Fig.(1). A resonance-like peak of $\chi_{\mathrm{Ni}}(T)$ is obtained for $T \gtrsim T_{C, \mathrm{Ni}}$ as has been reported previously [5]. With increasing interlayer coupling $J_{\text {inter }}$ the susceptibility peak is shifted to higher temperatures. Also, the maximum 
value of $\chi_{\mathrm{Ni}}(T)$ reduces strongly and its corresponding width increases markedly [5]. For a strong $J_{\text {inter }}$ the $\mathrm{Ni}$ susceptibility is so small that it may be hardly measurable. A singularity of $\chi_{\mathrm{Ni}}(T)$ will occur at $T=T_{C, \text { Co }}$ since there the induced Ni magnetization, although small, vanishes in accordance with the vanishing Co magnetization. Thus, $T_{C, \text { Co }}$ corresponds to the true phase transition temperature of the coupled magnetic $\mathrm{Co} / \mathrm{Cu} / \mathrm{Ni}$ trilayer system [5].

In Refs. [1]2] it was found for the $\mathrm{Co} / \mathrm{Cu} / \mathrm{Ni}$ trilayer system that the observed $\mathrm{Ni}$ remanent magnetization vanishes above a temperature $T_{\mathrm{Ni}}^{*}$, where $T_{C, \mathrm{Ni}}<T_{\mathrm{Ni}}^{*}<$ $T_{C, \text { Co. }}$. On the other hand, either no susceptibility signal or only a small peak in $\chi_{\mathrm{Ni}}(T)$ could be measured at $T_{\mathrm{Ni}}^{*}$. This might be due e.g. to the occurrence of a multidomain state or to a magnetic reorientation in the Ni film. A true phase transition in the thermodynamic sense is reminiscent to a nonanalytical behavior of the free energy, resulting in singularities of e.g. the correlation length or the susceptibility, as found at $T_{C, \mathrm{Co}}$. Regardless of the particular behavior of the magnetic properties at $T_{\mathrm{Ni}}^{*}$, we emphasize that the observed strong induced Ni magnetization and the shift of the Ni susceptibility for $T \gtrsim T_{C, \mathrm{Ni}}$ due to the interlayer coupling $J_{\text {inter }}$ is caused by the presence of magnetic fluctuations. To compare the measured and calculated temperature shift $\Delta T$ we have assumed $T_{\text {infl }} \approx T_{\mathrm{Ni}}^{*}$.

We have investigated asymmetric trilayers which exhibit different Curie temperatures for the decoupled $\mathrm{Ni}$ and Co films, e.g. for single magnetic layers or a thick nonmagnetic spacer. The interlayer coupling $J_{\text {inter }}$ influences mainly the magnetization of the $\mathrm{Ni}$ film, which has the lower ordering temperature, whereas $T_{C, \text { Co }}$ stays practically constant. On the other hand, a symmetric system, e.g. a $\mathrm{Ni} / \mathrm{Cu} / \mathrm{Ni}$ trilayer with equal $\mathrm{Ni}$ film thicknesses or a periodic multilayer system, has a single Curie temperature $T_{C}$. Here $J_{\text {inter }}$ will enhance $T_{C}$ considerably by amounts similar as discussed for the asymmetric trilayer. Indeed this has been observed for a Ni/Au multilayer system [4. In principle, by an appropriate combination of tri- and multilayers, for instance by varying the materials and the thicknesses of the magnetic and nonmagnetic layers, one might tune the magnetic properties according to possible applications.

In summary, we have calculated the action of the interlayer exchange coupling in a magnetic $\mathrm{Co} / \mathrm{Cu} / \mathrm{Ni}$ trilayer system by means of a many-body Green's function approach for a Heisenberg Hamiltonian. $J_{\text {inter }}$ induces a considerable magnetization in the Ni film for $T \gtrsim T_{C, \mathrm{Ni}}$, and shifts the Ni susceptibility peak to larger temperatures. Also the width of the Ni susceptibility increases, whereas its maximum value decreases strongly. We have shown that for reasonable values of $J_{\text {inter }}$ the observed strong induced Ni magnetization can be obtained only if the magnetic fluctuations in these $2 \mathrm{D}$ systems are taken into account properly. Corresponding results as calcu- lated by a MFT approach, which neglect these fluctuations, yield a 10 times smaller increase, and cannot explain the observed magnetic behavior for the $\mathrm{Co} / \mathrm{Cu} / \mathrm{Ni}$ trilayer system. The influence of the magnetic fluctuations becomes stronger for smaller Ni film thicknesses, s. Fig.(2), indicating the 2D character of the important correlations. Note that we have investigated the effect of $J_{\text {inter }}$ on the magnetic properties solely by considering thermal fluctuations. Other possible influences such as magnetic noncollinearities are not discussed here.

This work has been supported by the DFG, Sonderforschungsbereich 290. Discussions with C. Timm are gratefully acknowledged.

new address: Institut für Halbleiterphysik und Optik, Technische Universität Braunschweig, Mendelssohnstr. 3, D-38 106 Braunschweig, Germany.

[1] U. Bovensiepen, F. Wilhelm, P. Srivastava, P. Poulopoulos, M. Farle, A. Ney, and K. Baberschke, Phys. Rev. Lett. 81, 2368 (1998).

[2] A. Ney, F. Wilhelm, M. Farle, P. Poulopoulos, P. Srivastava, and K. Baberschke, Phys. Rev. B 59, R3938 (1999).

[3] P. Bruno and C. Chappert, Phys. Rev. Lett. 67, 1602 (1991).

[4] G. Bayreuther, F. Bensch, and V. Kottler, J. Appl. Phys. 79, 4509 (1996).

[5] R.W. Wang and D.L. Mills, Phys. Rev. B 46, 11681 (1992).

[6] S.V. Maleev, Sov. Phys. JETP 43, 1240 (1976); V.L. Pokrovsky and M.V. Feigel'man, ibid., 45, 291 (1977).

[7] D.A. Yablonsky, Phys. Rev. B 44, 4467 (1991); D. Kerkmann, D. Pescia, and R. Allenspach, Phys. Rev. Lett. 68, 686 (1992).

[8] S.V. Tyablikov, Ukr. Mat. Zh. 11, 287 (1959).

[9] H. Benson and D.L. Mills, Phys. Rev. 178, 839 (1969); P.J. Jensen, Ann. Physik 6, 317 (1997).

[10] P. Srivastava, F. Wilhelm, A. Ney, M. Farle, H. Wende, N. Haack, G. Ceballos, and K. Baberschke, Phys. Rev. B 58, 5701 (1998).

[11] S.V. Tyablikov, Methods in the quantum theory of magnetism, (Plenum Press, New York, 1967).

[12] H.B. Callen, Phys. Rev. 130, 890 (1963).

[13] C. Timm, S.M. Girvin, P. Henelius, and A.W. Sandvik, Phys. Rev. B 58, 1464 (1998).

[14] A. Ecker, P. Fröbrich, P.J. Jensen, and P.J. Kuntz, J. Phys.: Condens. Matter 11, 1557 (1999).

[15] In presence of the dipole coupling also the Green's functions $G_{i j}^{--(n)}$ should be taken into account in order to consider elliptical spin waves [e.g. T. Holstein and H. Primakoff, Phys. Rev. 58, 1098 (1940)]. The $G_{i j}^{--(n)}$ are neglected here because the dipole coupling is small as compared to the exchange coupling for the systems under consideration. We found that the results for the magnetic properties are not affected markedly by considering the $G_{i j}^{--(n)}$, for instance $T_{C}$ is reduced by $\sim 10 \%$. However, the computational effort enhances drastically.

[16] S.S.P. Parkin, Phys. Rev. Lett. 67, 3598 (1991). 